\title{
Aptidão física relacionada à saúde e fatores de risco predisponentes às doenças cardiovasculares em adolescentes*
}

\author{
Dartagnan P. Guedes \\ Joana E.R.P. Guedes \\ Decio S. Barbosa
}

Jair A. Oliveira

\author{
Universidade Estadual de Londrina \\ Paraná \\ Brasil
}

\section{RESUMO}

Recentemente, indicadores associados à aptidão física relacionada à saúde vêm sendo interpretados mediante informações referenciadas por critério. Informações referenciadas por critério direcionadas à saúde representam níveis desejáveis consistentes com reduzido risco de aparecimento e desenvolvimento de disfunções orgânicas que deverão ser alcançados pelos adolescentes. Objetivo do estudo foi analisar a validade de pontos de corte associados às informações referenciadas por critério preconizados pela proposta Physical Best na identificação de adolescentes portadores e não-portadores de fatores de risco predisponentes às doenças cardiovasculares. A amostra foi constituída por 281 adolescentes (157 moças e 124 rapazes) com idades entre 15 e 18 anos. Informações acerca da aptidão física relacionada à saúde foram obtidas mediante indicadores morfológicos (índice de massa corporal e somatório de espessuras das dobras cutâneas tricipital e subescapular) e resultados de testes motores (sentar-e-alcançar, abdominal modificado e caminhada/corrida de 1600 metros). Quanto aos fatores de risco predisponentes às doenças cardiovasculares, recorreu-se ao conteúdo de gordura corporal, ao consumo máximo de oxigênio, aos níveis de pressão arterial e às concentrações de lipídios-lipoproteínas plasmáticas. Estimativas quanto aos índices de sensibilidade, especificidade, valor preditivo positivo e eficiência foram utilizados para descrever a validade relativa. Os resultados mostraram que os índices de sensibilidade se apresentaram entre $20 \%$ e $70 \%$ e os de especificidade entre $30 \%$ e $85 \%$. Os indicadores morfológicos demonstraram que podem identificar corretamente três em cada quatro adolescentes com fatores de risco predisponentes às doenças cardiovasculares. Os pontos de corte associados aos resultados dos testes motores demonstraram baixos níveis de sensibilidade e elevada proporção de casos falso-positivos. Como conclusão, os achados sugerem que, independentemente dos pontos de corte utilizados, o índice de massa corporal e o somatório das espessuras das dobras cutâneas caracterizam-se como razoável alternativa para identificar a presença de fatores de risco predisponentes às doenças cardiovasculares em adolescentes.

Palavras-chave: aptidão física, fatores de risco, doenças cardio-vasculares, promoção da saúde, adolescentes.

\section{ABSTRACT \\ Health-related physical fitness and cardiovascular disease risk factors in adolescents}

Recently indicators associated with health-related physical fitness have been interpreted using criterion-referenced standards. A health-related criterion-referenced standard represents a desirable level to good health status and reduced disease risk that should be attainable by the adolescents. The present study was designed to evaluate the validity of the cut-off points associated with the Physical Best criterion-referenced standard in the identification of adolescents with and without cardiovascular disease risk factors. A total of 281 adolescents (157 girls and 124 boys) varying in age from 15 to 18 years was used in the study. Information on the health-related physical fitness was obtained by means of morphological indicators (body mass index and sum of two skinfolds: triceps and subscapular) and tests of motor performance (sitand-reach, sit-ups and mile run). Cardiovascular disease risk factors included body fat content, cardiorespiratory fitness, levels of arterial pressure and of plasma lipid-lipoprotein concentration. Estimates of sensitivity, specificity, positive predictive value and efficiency were used to describe the relative validity. The results showed that the sensitivity ranged approximately from $20 \%$ to $70 \%$, and the specificity ranged from $30 \%$ to $85 \%$. Morphological indicators may correctly identify three out of four adolescents with cardiovascular disease risk factors. Cut-off points associated with motor performance tests were characterized generally by low levels of sensitivity and corresponding high rates of false-negatives. In conclusion, the findings of study suggest that, independently of the cut-off points used, body mass index and sum of skinfold thickness were reasonable alternatives for cardiovascular disease risk factors screening in adolescents.

Keywords: physical fitness, risk factors, cardiovascular disease, health promotion, adolescence. 


\section{INTRODUÇÃO}

Indicadores associados à aptidão física têm sido tradicionalmente analisados e interpretados mediante confrontação com dados normativos, envolvendo referenciais idealizados com base em distribuição de percentis $(2,12,14)$. Parece evidente que análises com essas características tornam-se extremamente úteis quando a intenção é desenvolver comparações intra e inter-populacionais, o que permite visualização precisa quanto à magnitude de eventuais diferenças que possam surgir.

Abordagens desse tipo conduzem afirmações: (a) $50 \%$ dos rapazes com 14 anos de idade, analisados no município de Londrina - Paraná, não caminham/correm mais que $198 \mathrm{~m} / \mathrm{min}$ no teste de caminhada/corrida de longa distância; ou (b) enquanto por volta de $90 \%$ das moças norte-americanas com 17 anos de idade realizam até 36 repetições no teste abdominal, não mais que $50 \%$ das moças brasileiras apresentam resultado idêntico (21).

No entanto, como limitação, procedimentos de análise normativa não conseguem oferecer informações que possam contribuir na tentativa de esclarecer se resultados de indicadores associados à aptidão física efetivamente evidenciam níveis satisfatórios em relação à saúde. Desse modo, considerando que a opção desejada refere-se à aptidão física relacionada à saúde, questões fundamentais merecem maior atenção: (a) quão rápido rapazes de 14 anos devem caminhar/correr para comprovarem eficiência quanto à resistência cardiorrespiratória que possa traduzir níveis aceitáveis de saúde; ou (b) quantas repetições no teste abdominal são necessárias para moças de 17 anos demonstrarem níveis de força/resistência muscular satisfatórios em relação à saúde.

A princípio, mesmo assumindo importantes associações entre indicadores de aptidão física e condições de saúde em populações jovens $(9,33)$, análise equivalente às posições mais elevadas na distribuição de percentis não garante necessariamente níveis satisfatórios de saúde, na medida em que características da amostra da qual a distribuição de percentis foi derivada deverão afetar a capacidade de detecção das diferenças. Assim, a posição de escores individuais pode localizar-se no extremo superior da distribuição de percentis desenvolvida em segmento específico da população que possivelmente venha apresentar hábi- tos de vida inadequados para garantir condições de saúde satisfatórias, e, ao mesmo tempo e de forma antagônica, idênticos escores podem situar-se no extremo inferior quando confrontados com distribuição de percentis derivada com base em segmento da população que apresenta comportamentos favoráveis ao desenvolvimento de melhores condições de saúde. Com introdução de novos conceitos relacionados à aptidão física e à saúde assume-se que, quando as diferenças entre sujeitos deixam de ser importantes, análises referenciadas por critérios deverão apresentar vantagens em relação às confrontações com dados normativos (16). Neste caso, os critérios representam pontos de corte identificados com indicadores de aptidão física consistente com condições de saúde satisfatórias, independentemente da posição na distribuição de percentis. Dessa forma, ao recorrer as análises referenciadas por critérios, questão de interesse é identificar se cada jovem, individualmente, torna-se capaz de alcançar pontos de corte previamente estabelecidos em relação aos indicadores de aptidão física que possam assegurar algum grau de proteção diante do aparecimento e do desenvolvimento de disfunções hipocinéticas (9). Essência que procura justificar proposição de pontos de corte para indicadores de aptidão física relacionada à saúde baseia-se na premissa de que, para ocorrer redução na incidência de disfunções orgânicas, torna-se necessário alcançar níveis desejáveis quanto à quantidade de gordura corporal, à resistência cardiorrespiratória e ao desempenho musculoesquelético que possam conter eventual processo degenerativo induzido por hábitos de vida inadequados com relação à prática de atividades físicas (33).

Em oposição ao enfoque oferecido à análise referenciada por norma, onde o objetivo é apresentar escores equivalentes aos mais elevados valores de percentis, jovens que não alcançam pontos de corte previamente estabelecidos quanto aos indicadores da aptidão física relacionada à saúde deverão apresentar maior predisposição aos sintomas crônico-degenerativos, enquanto os que alcançam ou excedem aos pontos de corte estabelecidos demonstram menor risco nesse sentido. Assim, importante não é comparar um jovem com outros mediante valores normativos, mas sim, verificar se alcança o ponto de corte estabelecido em relação à saúde. 
Neste particular, a maior dificuldade encontrada pelos estudiosos da área constitui na determinação de escores associados aos indicadores de aptidão física que possam ser utilizados como ponto de corte, garantindo níveis desejados e absolutos necessários a melhor condição de saúde. Infelizmente, tudo indica que na atualidade não existe nenhum mecanismo confiável direcionado à proposição de pontos de corte que possa assegurar com alguma convicção níveis mínimos requeridos à redução dos riscos de disfunções degenerativas mediante indicadores de aptidão física.

Diante dessa situação incômoda, observa-se algumas iniciativas direcionadas à proposição de pontos de corte à partir de pesquisas experimentais, achados clínicos e designações arbitrárias baseadas em dados normativos $(3,4,15,35)$. Contudo, se entre diferentes propostas idealizadas existe consenso com relação às estratégias de ação quanto ao seu estabelecimento, desconhece-se qualquer tentativa de validação dos pontos de corte até então sugeridos.

Embora as manifestações clínicas associadas às doenças cardiovasculares surjam com maior freqüência durante a vida adulta, evidências científicas revelam que comprometimentos quanto à pressão arterial, aos níveis de lipídios-lipoproteínas plasmáticas, à disposição de gordura corporal e ao consumo máximo de oxigênio podem ter origem na adolescência (6). Em vista disso, torna-se possível supor que, mesmo não apresentando idêntico efeito sobre os índices de morbidade e mortalidade dos jovens, existe possibilidade de os fatores de risco que predispõem ao aparecimento e ao desenvolvimento de doenças cardiovasculares na fase adulta serem identificados já em idades precoces, constituindo-se portanto em importante referencial das condições de saúde dos adolescentes.

Diante dessa perspectiva, o objetivo do estudo foi analisar a validade de pontos de corte associados aos indicadores de aptidão física relacionada à saúde na identificação de adolescentes portadores e não-portadores de fatores de risco predisponentes às doenças cardiovasculares.

\section{MATERIAIS E MÉTODOS}

Para elaboração do estudo foram utilizadas informações contidas no banco de dados construído a partir do projeto de pesquisa "Atividade Física, Composição da Dieta e Fatores de Risco Predisponentes às Doenças Cardiovasculares em Adolescentes", desenvolvido entre agosto e novembro/1998, que inclui adolescentes entre 15 e 18 anos de idade de ambos os sexos.

O projeto de pesquisa teve como alvo escolares regularmente matriculados no ensino médio do Colégio de Aplicação ligado à Universidade Estadual de Londrina, Paraná, Brasil. Optou-se por envolver sujeitos que freqüentavam unicamente essa escola, por conta das características longitudinais do estudo (experimentação de programas de educação para saúde mediante intervenções dietéticas e de prática de exercícios físicos), e por sua representatividade no universo de escolares de ensino médio do município de Londrina, Paraná.

Os protocolos de intervenção no estudo foram aprovados pelo Comitê de Ética em Pesquisa da Universidade Estadual de Londrina e acompanham normas da Resolução 196/96 do Conselho Nacional de Saúde sobre pesquisa envolvendo seres humanos. A inclusão dos sujeitos no estudo ocorreu por desejo em participar do experimento e mediante autorização dos pais ou responsáveis. Para tanto, todos os escolares matriculados no ano letivo de 1998, juntamente com seus pais ou responsáveis, foram contatados e informados quanto à natureza e aos objetivos do estudo. Dos 313 escolares matriculados, 281 (90\%) concordaram em participar do estudo - tabela 1.

Tabela 1 - Número de sujeitos analisados no projeto "Atividade Física, Composição da Dieta e Fatores de Risco Predisponentes às Doenças Cardiovasculares em Adolescentes".

\begin{tabular}{lccc} 
Idade & Moças & Rapazes & Total \\
\hline 15 Anos & 33 & 25 & 58 \\
16 Anos & 48 & 37 & 85 \\
17 Anos & 41 & 34 & 75 \\
18 Anos & 35 & 28 & 63 \\
$15-18$ Anos & 157 & 124 & 281
\end{tabular}

Como informação adicional da amostra analisada no estudo, destaca-se que, com base nos critérios de classificação socioeconômica das famílias dos escolares, mediante informações quanto ao nível de escola- 
ridade do chefe da família, às condições de moradia, à posse de utensílios domésticos, automóveis e número de empregados domésticos (1), observou-se que $25 \%$ dos escolares foram categorizados em menor nível socioeconômico, 18 \% em maior nível e $57 \%$ em níveis intermediários. Com relação aos critérios de classificação de maturação sexual sugeridos por Tanner (39), 12\% das moças analisadas apresentavam desenvolvimento mamário equivalente ao estágio III, $60 \%$ ao estágio IV e $28 \%$ ao estágio V. Entre os rapazes, $47 \%$ se encontravam no estágio IV de desenvolvimento de pilosidade pubiana, e os $53 \%$ restantes no estágio $\mathrm{V}$.

Informações acerca da aptidão física relacionada à saúde foram obtidas mediante indicadores morfológicos e de desempenho motor, acompanhando orienta- ções descritas por intermédio da proposta Physical Best (3). Como indicadores morfológicos utilizaram-se o índice de massa corporal (IMC) e o somatório dos valores de espessura das dobras cutâneas medidas nas regiões tricipital e subescapular ( $\left.\sum E D C\right)$. Com relação ao desempenho motor, foram incluídos resultados dos testes motores "sentar-e-alcançar" (SEAL), abdominal modificado (ABDO) e caminhada/corrida de 1600 metros (CORR). A definição dos pontos de corte associados aos indicadores morfológicos e de desempenho motor voltados à aptidão física relacionada à saúde, sugeridos na proposta Physical Best, é apresentada na tabela 2 . Ao consultar a literatura, percebe-se que esses pontos de corte são os que têm recebido maior aceitação, tendo sido empregados em vários outros estudos $(17,21,28,40,41)$.

Tabela 2 - Pontos de corte associados aos indicadores de aptidão física relacionada à saúde sugeridos mediante proposta Physical Best.

\begin{tabular}{|c|c|c|c|c|c|c|c|c|}
\hline & \multicolumn{2}{|c|}{15 Anos } & \multicolumn{2}{|c|}{16 Anos } & \multicolumn{2}{|c|}{ 17 Anos } & \multicolumn{2}{|c|}{18 Anos } \\
\hline & Moças & Rapazes & Moças & Rapazes & Moças & Rapazes & Moças & Rapazes \\
\hline IMC $\left(\mathrm{Kg} / \mathrm{m}^{2}\right)$ & $17-24$ & $17-24$ & $17-24$ & $17-24$ & $17-24$ & $18-25$ & $18-25$ & $18-25$ \\
\hline$\sum \mathrm{EDC}(\mathrm{mm})$ & $16-36$ & $12-25$ & $16-36$ & $12-25$ & $16-36$ & $12-25$ & $16-36$ & $12-25$ \\
\hline SEAL (cm) & 25 & 25 & 25 & 25 & 25 & 25 & 25 & 25 \\
\hline ABDO (rep) & 35 & 42 & 35 & 44 & 35 & 44 & 35 & 44 \\
\hline CORR (min) & $10: 30$ & $7: 30$ & $10: 30$ & $7: 30$ & $10: 30$ & 7:30 & $10: 30$ & 7:30 \\
\hline
\end{tabular}

Adaptado de American Alliance for Health, Physical Education, Recreation and Dance [1988]

Com referência aos fatores de risco predisponentes às doenças cardiovasculares (DCV), recorreu-se as informações voltadas à pressão arterial em repouso, ao teor sangüíneo de lipídios circulantes, às estimativas da quantidade de gordura relativa ao peso corporal e ao consumo máximo de oxigênio.

Os níveis de pressão arterial sistólica (PAS) e diastólica (PAD) foram aferidos com auxílio de esfigmomanômetro de coluna de mercúrio. Com o adolescente sentado, após um período mínimo de 5 minutos de repouso, a pressão arterial foi medida no braço esquerdo. O valor da PAS correspondeu à fase I de Korotkoff e o da PAD à fase V, ou de desaparecimento dos sons. Foram realizadas duas medidas, sendo que o valor médio de ambas foi considerado para efeito de análise.

Dosagens dos lipídios plasmáticos foram realizadas mediante coleta de amostras de $10 \mathrm{ml}$ de sangue venoso na prega do cotovelo, após um período de 10$12 \mathrm{~h}$ em jejum, entre 07:00 e 08:00h da manhã. Soro foi imediatamente separado por centrifugação, sendo determinados os teores de triglicerídios (TG), colesterol total (CT) e frações, lipoproteínas de baixa densidade (LDL-C) e de alta densidade (HDL-C).

Determinou-se o colesterol sérico total pelo método enzimático colesterol oxidase/peroxidase em aparelho espectofotômetro. O HDL-C foi medido pelo método reativo precipitante, e o LDL-C calculado pela fórmula de Friedewald (20). Os triglicerídios séricos foram determinados pelo método enzimático glicerol. 
A proporção de gordura em relação ao peso corporal (GORD) foi estimada a partir dos valores de espessura de dobras cutâneas determinadas nas regiões tricipital e subescapular, mediante utilização de equações preditivas específicas para sexo, idade, grupo racial e maturação biológica idealizadas por Slaughter et al. (38). O consumo máximo de oxigênio $\left(\mathrm{VO}_{2} \mathrm{max}\right)$ foi estimado por intermédio de teste de esforço de carga máxima em esteira rolante, de acordo com protocolo de Bruce et al. (11). O tempo necessário para alcançar a freqüência cardíaca máxima teórica (220 - idade) foi utilizado para estabelecer estimativas do $\mathrm{VO}_{2}$ max. Para aqueles adolescentes que não foram capazes de alcançar sua freqüência cardíaca máxima teórica, foi empregado como referencial a duração do teste de esforço.

Para análise conjunta dos fatores de risco predisponentes às DCV, optou-se por estabelecer único escore para cada adolescente. Para tanto, considerando separadamente os diferentes fatores de risco considerados: PAD, PAS, CT, HDL, LDL, TG, GORD e $\mathrm{VO}_{2}$ max, a amostra envolvida no estudo foi ajustada de acordo com a distribuição de quintis. Para efeito de interpretação, atribuiu-se valor 1 para os fatores de risco reunidos no quintil inferior, valor 2 aos fatores de risco agrupados no segundo quintil, e assim por diante, de tal forma que aos fatores de risco localizados no quintil mais elevado foi atribuído valor 5. O escore associado ao conjunto dos fatores de risco foi estabelecido mediante somatório dos valores atribuídos aos quintis de cada um dos fatores de risco separadamente.

Logo, o escore mínimo possível do conjunto de fatores de risco tornou-se igual a 8 (valor 1 correspondente ao quintil mais baixo x 8 fatores de risco) e o escore máximo possível igual a 40 (valor 5 correspondente ao quintil mais elevado $\mathrm{x} 8$ fatores de risco). Em ambos os sexos, classificou-se adolescentes como portadores de fatores de risco predisponentes às DCV quando o somatório dos valores atribuídos a cada um dos fatores de risco se apresentou igual ou superior a 34, ou seja, equivalente ao quintil mais elevado na distribuição do conjunto dos fatores de risco.

A validade dos pontos de corte associados aos indicadores morfológicos e de desempenho motor voltados à aptidão física relacionada à saúde, sugeridos pela proposta Physical Best, na tentativa de identificar adolescentes portadores e não-portadores de conjunto de fatores de risco predisponentes às DCV, foi analisada mediante propriedades de sensibilidade, especificidade, valor preditivo positivo e eficiência tabela 3 .

Tabela 3 - Delineamento de validação dos indicadores de aptidão física relacionados à saúde na identificação de adolescentes portadores de fatores de risco predisponentes às doenças cardiovasculares.

Pontos de Corte dos Indicadores de Aptidão Física

Não Atendem os Ponto de Corte

Atendem os Ponto de Corte

Total
Conjunto de Fatores de Risco Predisponentes às Doenças Cardiovasculares

Presente Ausente

A Verdadeiro- Positivo C

Falso-Negativo $A+C$

Falso-Positivo

$$
\text { D }
$$

Verdadeiro-Negativo

$$
\mathrm{B}+\mathrm{D}
$$

Total $A+B$

Sensibilidade $[\%]=(A / A+C) 100 ;$ Especificidade $[\%]=(D / B+D) 100 ;$ Valor Preditivo Positivo $[\%]=(A / A+B) 100 ;$ Eficiência $[\%]=(A+D / A+B+C+D) 100$

Nesse contexto, a sensibilidade representa a proporção de adolescentes classificados como portadores de fatores de risco predisponentes às DCV que não atendem os pontos de corte sugeridos para indicado- res de aptidão física relacionada à saúde (casos verdadeiro-positivos). A especificidade corresponde à proporção de adolescentes classificados como não-portadores de fatores de risco predisponentes às 
DCV que atendem os pontos de corte sugeridos para indicadores de aptidão física relacionada à saúde (casos verdadeiro-negativos). O valor preditivo positivo é definido como a probabilidade dos adolescentes serem classificados como portadores de fatores de risco quando não atendem os pontos de corte sugeridos para indicadores de aptidão física relacionada à saúde. A eficiência é entendida como a proporção de adolescentes classificados como portadores de fatores de risco predisponentes às DCV que não atendem os pontos de corte sugeridos para indicadores de aptidão física relacionada à saúde e os adolescentes classificados como não-portadores de fatores de risco predisponentes às DCV que atendem os pontos de corte sugeridos para indicadores de aptidão física relacionada à saúde.

Foram estabelecidos também, índices de probabilidade de ocorrência positiva (sensibilidade / 1 - especificidade) e negativa ( 1 - sensibilidade / especificidade). O índice de probabilidade de ocorrência positiva deverá oferecer informações quão mais provável é identificar adolescentes como portadores de fatores de risco predisponentes às DCV quando esses não se ajustam aos pontos de corte associados aos indicadores de aptidão física relacionada à saúde. Em contrapartida, índices de probabilidade de ocorrência negativa deverão oferecer informações quão mais provável é identificar adolescentes como não-portadores de fatores de risco predisponentes às DCV quando esses se ajustam aos pontos de corte assumidos para os indicadores de aptidão física relacionada à saúde. Ocorrência de casos falso-positivos e falso-negativos deverá constituir propriedades indesejadas no processo de validação dos pontos de corte associados à aptidão física relacionada à saúde: proporção de adolescentes que não atenderam os pontos de corte sugeridos e que foram classificados como não-portadores de fatores de risco predisponentes às DCV; proporção de adolescentes que atenderam os pontos de corte sugeridos e que foram classificados como portadores de fatores de risco predisponentes às DCV. Mediante procedimentos de análise da curva ROC (Receiver Operating Characteristic) foram estabelecidos escores para os indicadores de aptidão física relacionada à saúde correspondente ao melhor ajuste estatístico entre casos verdadeiro-positivos (sensibilidade) e falso-positivos ( 1 - especificidade) na classifi- cação de adolescentes portadores de fatores de riscos predisponentes às DCV. Em tese, escores indicados por intermédio da análise da curva ROC constituem-se em pontos de corte que deverão minimizar ocorrência de casos falso-positivos e falso-negativos, sugerindo portanto mais adequado equilíbrio entre sensibilidade e especificidade para os dados disponíveis (43).

Recorreu-se as informações provenientes da área sob a curva ROC para estabelecer níveis de precisão dos escores associados aos indicadores de aptidão física relacionada à saúde que apresentam mais adequado equilíbrio entre casos verdadeiro-positivos e falso-positivos na classificação dos adolescentes portadores de fatores de risco predisponentes às DCV (24). Com relação a sua interpretação, assumiu-se que, quando não se conseguiu, nos grupos de adolescentes que atenderam e não-atenderam os pontos de corte, distinguir entre aqueles que foram classificados como portadores e não-portadores de fatores de risco predisponentes às $\mathrm{DCV}$, a área sob a curva ROC deverá se apresentar por volta de 0,5. Quando existiu perfeita distinção entre as duas condições analisadas - (a) atendimento dos pontos de corte não sendo portadores de fatores de risco; e (b) não-atendimento dos pontos de corte sendo portadores de fatores de risco - a área sob a curva ROC deverá se aproximar de 1,0 .

Diferenças entre ambos os sexos quanto aos indicadores de aptidão física relacionada à saúde e aos fatores de risco predisponentes às DCV foram detectadas mediante teste " $t$ " de Student para dados não-emparelhados. $\mathrm{O}$ tratamento estatístico das informações foi realizado mediante pacote computadorizado Computer Program for Statistics in Medicine (31).

\section{RESULTADOS}

Informações estatísticas com relação às variáveis analisadas são mostradas na tabela 4. No que se refere aos indicadores de aptidão física relacionada à saúde, resultados associados ao $\sum$ EDC demonstram nítida tendência entre moças em acumular maiores quantidades de gordura subcutânea. No entanto, quando da comparação dos valores médios relativos ao IMC, não se encontram diferenças entre os sexos que possam ser apontadas estatisticamente. Quanto aos resultados dos testes de desempenho motor, 
confirmando evidências apresentadas na literatura $(22,29)$, os rapazes apresentam valores médios significativamente superiores no ABDO e no CORR. Os resultados médios encontrados no SEAL apontam diferenças estatísticas favoráveis as moças.

Tabela 4 - Valores de média, desvio-padrão e estatísticas "t" quanto às informações associadas aos indicadores de aptidão física relacionada à saúde e aos fatores de risco predisponentes às doenças cardiovasculares.

\begin{tabular}{|c|c|c|c|c|}
\hline & $\begin{array}{c}\text { Moças } \\
(n=157)\end{array}$ & $\begin{array}{l}\text { Rapazes } \\
(n=124)\end{array}$ & $\begin{array}{c}\text { Teste } \\
\text { " } \mathrm{t} \text { " }\end{array}$ & $\mathrm{p}<\mathrm{t}$ \\
\hline \multicolumn{5}{|l|}{ APTIDÃO FÍ́SICA } \\
\hline IMC $\left(\mathrm{kg} / \mathrm{m}^{2}\right)$ & $21,11 \pm 2,81$ & $21,37 \pm 3,17$ & 0,744 & ns \\
\hline$\sum \operatorname{EDC}(\mathrm{mm})$ & $32,06 \pm 9,28$ & $22,22 \pm 9,60$ & 8,680 & 0,000 \\
\hline SEAL $(\mathrm{cm})$ & $33,55 \pm 6,87$ & $29,37 \pm 9,51$ & 3,502 & 0,001 \\
\hline ABDO (rep) & $34,22 \pm 7,41$ & $42,91 \pm 7,34$ & 8,047 & 0,000 \\
\hline CORR (min) & $11,02 \pm 1,33$ & $8,00 \pm 1,34$ & 15,221 & 0,000 \\
\hline \multicolumn{5}{|l|}{ FATORES DE RISCO } \\
\hline $\operatorname{PAD}(\mathrm{mmHg})$ & $71,41 \pm 8,61$ & $73,86 \pm 7,91$ & 1,454 & $\mathrm{~ns}$ \\
\hline PAS (mmHg) & $109,47 \pm 9,56$ & $118,63 \pm 10,20$ & 7,727 & 0,000 \\
\hline CT $(\mathrm{mg} / \mathrm{dl})$ & $146,23 \pm 23,36$ & $134,32 \pm 23,21$ & 4,071 & 0,000 \\
\hline HDL-C (mg/dl) & $52,52 \pm 12,14$ & $46,44 \pm 6,77$ & 4,749 & 0,000 \\
\hline LDL-C (mg/dl) & $77,70 \pm 22,66$ & $72,14 \pm 20,88$ & 2,020 & 0,044 \\
\hline $\mathrm{TG}(\mathrm{mg} / \mathrm{dl})$ & $80,00 \pm 35,71$ & $81,49 \pm 37,17$ & 0,326 & ns \\
\hline GORD [\%] & $26,72 \pm 5,56$ & $15,99 \pm 8,71$ & 12,541 & 0,000 \\
\hline $\mathrm{VO}_{2} \max (\mathrm{ml} / \mathrm{kg} / \mathrm{min})$ & $40,92 \pm 5,49$ & $50,88 \pm 7,71$ & 12,597 & 0,000 \\
\hline
\end{tabular}

Com relação aos fatores de risco predisponentes às $\mathrm{DCV}$, constata-se valores médios referentes à pressão arterial sistólica significativamente mais elevados entre rapazes. Quanto aos teores plasmáticos de lipídios/lipoproteinas, os resultados apontam valores médios estatisticamente mais elevados entre as moças na maioria dos componentes analisados. As concentrações médias de TG mostram ser semelhantes entre os sexos. Estimativas quanto à GORD demonstram diferenças estatísticas favoráveis as moças $(26,72$ vs $15,99 \%)$, sugerindo um índice de dimorfismo sexual próximo de $40 \%$; enquanto estimativas associadas ao $\mathrm{VO}_{2}$ max apontam vantagens estatisticamente significativas a favor dos rapazes ( 50,88 vs $40,92 \mathrm{ml} / \mathrm{kg} / \mathrm{min})$, apontando um índice de dimorfismo sexual por volta de $20 \%$. Os comportamentos e magnitudes das diferenças inter-sexos relacionados aos fatores de risco predisponentes às DCV observadas no estudo coincidem com tendên- cias encontradas na literatura $(5,36,37)$.

As proporções de adolescentes analisados no estudo que atenderam pontos de corte sugeridos na proposta Physical Best associados aos indicadores de aptidão física relacionada à saúde são apresentadas na figura 1. Constata-se que o maior comprometimento foi observado nos resultados do CORR $(56 \%$ das moças e $76 \%$ dos rapazes) e do ABDO (60\% em ambos os sexos). Quanto aos resultados do SEAL, verifica-se que uma menor proporção de adolescentes deixaram de atender os pontos de corte sugeridos ( $11 \%$ e $30 \%$ das moças e dos rapazes, respectivamente). Informações referentes aos indicadores morfológicos da aptidão física relacionada à saúde demonstram que $16 \%$ das moças e $26 \%$ dos rapazes apresentaram IMC não-adequados para a idade, e por volta de $31-32 \%$ dos adolescentes de ambos os sexos apresentaram $\sum$ EDC não-ajustados aos pontos de corte preconizados. 


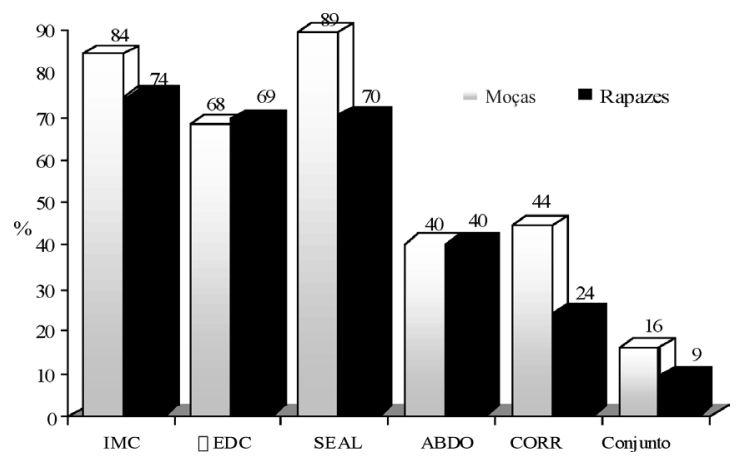

Figura 1 - Proporção de adolescentes que atenderam os pontos de corte associados aos indicadores de aptidão física relacionada à saúde sugeridos a partir da proposta Physical Best.

Considerando que os adolescentes poderiam atender ponto de corte conferido a determinado indicador, e não a outro, visto que cada um dos cinco indicadores contemplados na proposta Physical Best acompanha prioritariamente diferentes componentes da aptidão física relacionada à saúde, procurou-se determinar a proporção de adolescentes que alcançaram simultaneamente pontos de corte estabelecidos para os múltiplos componentes. Em ambos os sexos, os resultados apontam alarmante diminuição na proporção de adolescentes que apresentaram níveis satisfatórios de aptidão física relacionada à saúde. Não mais que $16 \%$ das moças e $9 \%$ dos rapazes atenderam as exigências de desempenho motor e, simultaneamente, apresentaram IMC e $\sum$ EDC ajustados aos pontos de corte estabelecidos a partir da proposta Physical Best. Mediante figura 2 visualiza-se a proporção de adolescentes que apresentaram comprometimento nos fatores de risco predisponentes às DCV. Acompanhando valores de referência para idade e sexo sugeridos na literatura (7), verifica-se que uma proporção significativa dos adolescentes analisados no estudo apresentaram elevado risco predisponente às DCV devido à excessiva quantidade de gordura corporal ( $27 \%$ das moças e $25 \%$ dos rapazes) e ao menor $\mathrm{VO}_{2} \max (14 \%$ e $8 \%$ das moças e dos rapazes, respectivamente). Concentrações de lipídios-lipoproteínas plasmáticas associadas ao aumento do risco de DCV foram comuns em aproximadamente $9 \%$ das moças e $6 \%$ dos rapazes. Quanto à pressão arterial, por volta de $13 \%$ das moças e $10 \%$ dos rapazes apresentaram valores acima dos limites esperados.

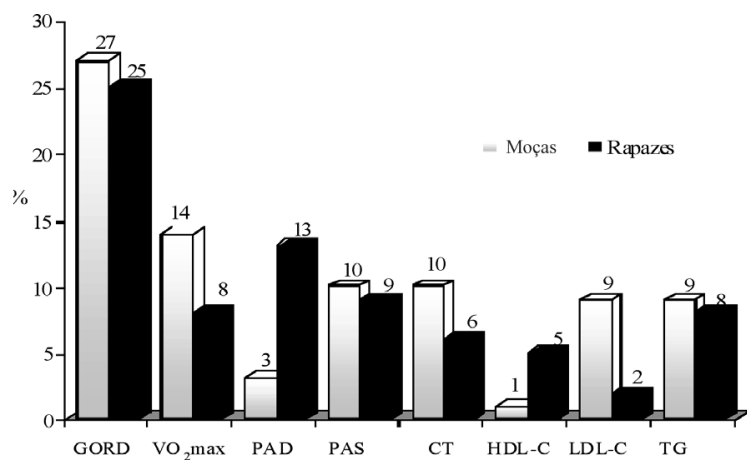

Figura 2 - Proporção de adolescentes identificados como portadores de fatores de risco predisponentes às doenças cardiovasculares.

Com relação à proporção de adolescentes que apresentaram múltiplos fatores de risco, $28 \%$ das moças e $26 \%$ dos rapazes demonstraram ser portadores de pelo menos um fator de risco associado ao aparecimento e ao desenvolvimento de DCV.

Concomitância de dois ou três fatores de risco estava presente, respectivamente, em $15 \%$ e $4 \%$ das moças, e $13 \%$ e $2 \%$ dos rapazes.

Informações quanto à validade estatística dos pontos de corte sugeridos na proposta Physical Best associados aos indicadores de aptidão física relacionada à saúde para identificação de adolescentes portadores e não-portadores de fatores de risco predisponentes às DCV são apresentadas na tabela 5 . Verifica-se que, tanto nos indicadores morfológicos como nos de desempenho motor, os índices de sensibilidade se mostram discretamente mais elevados entre os rapazes, enquanto os índices de especificidade apresentados pelas moças se destacam em relação aos dos rapazes. 
Tabela 5 - Estatística de validação dos pontos de corte sugeridos mediante proposta Physical Best associados aos indicadores de aptidão física relacionada à saúde para identificação de adolescentes portadores e não-portadores de fatores de risco predisponentes às doenças cardiovasculares.

\begin{tabular}{|c|c|c|c|c|c|c|c|c|}
\hline & & & \multicolumn{6}{|c|}{ VALOR PREDITIVO } \\
\hline & \multicolumn{2}{|c|}{ Sensibilidade } & \multicolumn{2}{|c|}{ Especificidade } & \multicolumn{2}{|c|}{ Positivo } & \multicolumn{2}{|c|}{ Eficiência } \\
\hline & Moças & Rapazes & Moças & Rapazes & Moças & Rapazes & Moças & Rapazes \\
\hline IMC & 49 & 50 & 89 & 84 & 50 & 48 & 76 & 67 \\
\hline$\sum E D C$ & 63 & 69 & 85 & 78 & 47 & 53 & 74 & 80 \\
\hline SEAL & 22 & 25 & 69 & 49 & 25 & 18 & 50 & 49 \\
\hline ABDO & 23 & 33 & 55 & 39 & 14 & 22 & 47 & 56 \\
\hline CORR & 48 & 50 & 45 & 27 & 25 & 25 & 48 & 49 \\
\hline
\end{tabular}

Com relação ao IMC, em ambos os sexos, observam-se índices de sensibilidade próximos de $50 \%$, o que assegura uma proporção de 2:1 na ocorrência de casos falso-negativos (100 - sensibilidade). Em contrapartida, constata-se uma elevada especificidade (89\% e $84 \%$ para moças e rapazes, respectivamente), sugerindo menor incidência de casos falso-positivos (100 - especificidade). Dessa forma, se, por um lado, parece existir grande probabilidade dos adolescentes que se ajustaram aos pontos de corte sugeridos para IMC serem identificados como não-portadores de fatores de risco predisponentes às DCV, por outro, existe menor probabilidade dos adolescentes que não se ajustaram aos pontos de corte serem identificados como portadores de fatores de risco.

No que se refere ao $\sum E D C$, verifica-se que, em ambos os sexos, os pontos de corte sugeridos mediante proposta Physical Best elevam a capacidade de identificar adolescentes portadores de fatores de risco predisponentes às DCV, apontando índices de sensibilidade por volta de $63-69 \%$, sem no entanto, alterar de maneira significativa os índices de especificidade ( $85 \%$ entre moças e $78 \%$ entre rapazes). Ao analisar o comportamento quanto aos valores preditivos positivos, comprova-se que, em ambos os sexos, os índices encontrados associados ao IMC e ao $\sum$ EDC se aproximam de $50 \%$. Em assim sendo, assume-se que, entre aqueles adolescentes que não atenderam os pontos de corte para indicadores morfológicos da aptidão física relacionada à saúde, apenas metade deles foram identificados como portadores de fatores de risco predisponentes às DCV. Quanto à propriedade de eficiência, visualizam-se também discretas diferenças entre os índices observados quando do envolvimento do IMC e do $\sum$ EDC.
De maneira geral, os índices de eficiência encontrados foram razoavelmente elevados $(74-76 \%$ entre as moças e $67-80 \%$ entre os rapazes).

Com relação às estatísticas de validação dos pontos de corte associados aos indicadores de desempenho motor da aptidão física relacionada à saúde, verifica-se que, em comparação com índices encontrados para indicadores morfológicos, esses são consideravelmente menores em ambos os sexos. Mais elevado índice de sensibilidade foi observado no CORR ( $48 \%$ e $50 \%$ entre moças e rapazes, respectivamente), enquanto que o SEAL e o ABDO apresentaram muito baixa proporção de casos verdadeiro-positivos (22-23\% em moças e $25-33 \%$ em rapazes). Em vista disso, a probabilidade de ocorrência de casos falso-negativos (100 - sensibilidade) torna-se preocupantemente elevada, indicando que, de cada quatro adolescentes identificados como portadores de fatores de risco predisponentes às DCV, aproximadamente dois deles no CORR e três no SEAL e no ABDO são capazes de atender os pontos de corte sugeridos na proposta Physical Best.

Os índices de especificidade equivalentes aos pontos de corte associados aos indicadores de desempenho motor foram mais elevados entre moças, apesar de acentuadamente mais baixos que os encontrados quando da análise dos indicadores morfológicos. Entre $45-69 \%$ das moças e $27-49 \%$ dos rapazes, não identificados como portadores de fatores de risco predisponentes às $\mathrm{DCV}$, atenderam pontos de corte preconizados para indicadores de desempenho motor. Conseqüentemente, as proporções de casos falso-positivos (100 - especificidade) tornam-se excessivamente elevadas. 
A análise dos valores preditivos positivos encontrados aponta que, não mais de $25 \%$ dos adolescentes que não atenderam pontos de corte equivalentes ao desempenho motor foram identificados como portadores de fatores de risco predisponentes às DCV, comprometendo portanto, eventual tentativa de envolvimento dos pontos de corte sugeridos na proposta Physical Best na identificação de adolescentes portadores de fatores de risco predisponentes às DCV. De maneira similar, os índices de eficiência encontrados nos três indicadores de desempenho motor da aptidão física relacionada à saúde revelam baixos níveis de acurácia na identificação de casos verdadei- ros em ambos os sexos (49-56\%). Esses resultados sugerem que, de cada 2 adolescentes considerados na amostra, apenas 1 foi corretamente classificado como verdadeiro-positivo ou verdadeiro-negativo. $\mathrm{Na}$ tentativa de maximizar o ajuste estatístico na ocorrência de casos positivos, e, conseqüentemente, aprimorar a eficiência dos indicadores de aptidão física relacionada à saúde na identificação de adolescentes portadores e não-portadores de fatores de risco predisponentes às DCV, a tabela 6 apresenta informações associadas à validação dos pontos de corte estabelecidos pela curva ROC, com base nos dados reunidos no presente estudo.

Tabela 6 - Estatística de validação dos pontos de corte associados aos indicadores de aptidão física relacionada à saúde sugeridos pela curva ROC com base em amostra de adolescentes do município de Londrina - Paraná na identificação de fatores de risco predisponentes às doenças cardiovasculares.

\begin{tabular}{|c|c|c|c|c|c|c|c|c|}
\hline & & & & & VALOF & DITIVO & & \\
\hline & $\mathrm{Se}$ & lidade & Esp & idade & & & & ncia \\
\hline & Moças & Rapazes & Moças & Rapazes & Moças & Rapazes & Moças & Rapazes \\
\hline IMC & 54 & 53 & 81 & 85 & 48 & 49 & 75 & 76 \\
\hline$\sum E D C$ & 60 & 73 & 86 & 82 & 57 & 56 & 80 & 81 \\
\hline SEAL & 32 & 31 & 77 & 54 & 14 & 16 & 51 & 50 \\
\hline ABDO & 42 & 56 & 83 & 56 & 44 & 26 & 53 & 44 \\
\hline CORR & 73 & 58 & 81 & 56 & 22 & 17 & 31 & 40 \\
\hline
\end{tabular}

Os pontos de corte equivalentes ao IMC (16-23 $\mathrm{kg} / \mathrm{m}^{2}$ entre moças e $16-22 \mathrm{~kg} / \mathrm{m}^{2}$ entre rapazes) e ao $\sum E D C$ (16-34 $\mathrm{mm}$ e $11-24 \mathrm{~mm}$ para moças e rapazes, respectivamente), correspondentes a otimização do equilíbrio sensibilidade-especificidade, mostram-se menores que os originalmente sugeridos na proposta Physical Best. Com novos pontos de corte, a probabilidade de ocorrência de casos falsos-positivos diminui discretamente. A princípio, essa redução já era esperada, na medida em que, pontos de corte mais exigentes tendem a aumentar concomitantemente os índices de sensibilidade e especificidade, reduzindo portanto a probabilidade de ocorrência de casos falso-negativos e falso-positivos. Quanto aos indicadores de desempenho motor asso- ciados à aptidão física relacionada à saúde, constatam-se importantes diferenças entre os pontos de corte apontados pela curva ROC e aqueles sugeridos mediante a proposta Physical Best. No que se refere ao SEAL, enquanto a proposta Physical Best sugere resultados de $25 \mathrm{~cm}$ como ponto de corte para ambos os sexos, dados analisados no presente estudo apontam maior ocorrência de casos positivos ao assumir um ponto de corte mais elevado: 29 e $32 \mathrm{~cm}$ para moças e rapazes, respectivamente. Um ajuste estatístico mais adequado para o ABDO ocorre quando o ponto de corte é estabelecido em 28 repetições para moças e 41 repetições para rapazes, ou seja, abaixo do ponto de corte originalmente sugerido: 35 e 44 repetições, respectivamente. Com rela- 
ção ao CORR, entre moças, melhor adequação quanto às estatísticas de validação ocorreu ao considerar o ponto de corte de 9:50 minutos, ao invés de 10:30 como sugere a proposta Physical Best. Em contrapartida, entre rapazes, faz-se necessário elevar o resultado inicialmente proposto de 7:30 para 7:50 minutos. Apesar dos pontos de corte voltados ao desempenho motor, determinados mediante análise da curva ROC, terem oferecido índices de sensibilidade e de especificidade mais elevados que os pontos de corte previamente sugeridos na proposta Physical Best, e, por sua vez, menor probabilidade de ocorrência de casos falso-positivos e falso-negativos, ambos os grupos de pontos de corte apresentam índices de eficiência similares, sugerindo apenas discretas vantagens na capacidade discriminatória entre adolescentes portadores e não-portadores de fatores de risco predisponentes às DCV. Ainda, mediante novos pontos de corte, a probabilidade de identificação inadequada de adolescentes não-portadores de fatores de risco predisponentes às DCV que não atenderam os pontos de corte (100 - especificidade) foi menor que a probabilidade de identificação inadequada de adolescentes portadores de fatores de risco predisponentes às DCV que atenderam os pontos de corte voltados ao desempenho motor direcionado à aptidão física relacionada à saúde (100 - sensibilidade), sobretudo entre moças.

Estimativas quanto à área sob curva ROC com relação aos pontos de corte associados aos indicadores de aptidão física relacionada à saúde na identificação de adolescentes portadores de fatores de risco predisponentes às DCV, apontadas com base na amostra analisada no presente estudo, são apresentadas na tabela 7. De maneira geral, não apresentando diferenças significativas entre sexos, constata-se que as áreas sob curvas ROC associadas aos indicadores morfológicos são mais elevadas que as apresentadas pelos indicadores de desempenho motor. Dessa forma, novos pontos de corte propostos para IMC e $\sum$ EDC poderão distinguir igualmente e com razoável precisão adolescentes portadores e não-portadores de fatores de risco predisponentes às DCV. Ambos os indicadores morfológicos apresentam 76-79\% de chance de identificar corretamente a presença de fatores de risco predisponentes às DCV nos adolescentes analisados no estudo.
Tabela 7 - Características da área sob a curva ROC (média, desvio-padrão e intervalos de confiança] com relação aos pontos de corte associados aos indicadores de aptidão física relacionada à saúde sugeridos com base em amostra de adolescentes do município de Londrina - Paraná na identificação de fatores de risco predisponentes às doenças cardiovasculares.

\begin{tabular}{lcc} 
& \multicolumn{1}{c}{ Moças } & Rapazes \\
\hline IMC & $0,76 \pm 0,051(0,68-0,83)$ & $0,77 \pm 0,058(0,69-0,84)$ \\
$\sum$ EDC & $0,76 \pm 0,051(0,68-0,82)$ & $0,79 \pm 0,056(0,71-0,86)$ \\
SEAL & $0,52 \pm 0,066(0,42-0,62)$ & $0,53 \pm 0,068(0,43-0,62)$ \\
ABDO & $0,54 \pm 0,058(0,44-0,62)$ & $0,55 \pm 0,056(0,45-0,64)$ \\
CORR & $0,51 \pm 0,061(0,41-0,61)$ & $0,51 \pm 0,065(0,41-0,61)$
\end{tabular}

Entre os indicadores de desempenho motor, a capacidade dos pontos de corte melhores ajustados para identificar corretamente adolescentes portadores de fatores de risco predisponentes às DCV é baixa em ambos os sexos, apresentando área sob curva ROC próxima de 0,50. Em assim sendo, os achados apresentados no presente estudo mostram que os resultados de testes motores, associados à aptidão física relacionada à saúde, não se definem como atributos que possam identificar com precisão aceitável fatores de risco predisponentes às $\mathrm{DCV}$. Os resultados dos três testes motores apresentaram por volta de $50 \%$ de chance de identificar corretamente a presença de fatores de risco predisponentes às DCV nos adolescentes analisados no estudo.

\section{DISCUSSÃO}

A adoção de hábitos dietéticos hiperfágicos e com elevada proporção de alimentos ricos em gorduras, acompanhados por menores níveis de prática habitual de atividade física, vem contribuindo significativamente para o aparecimento e desenvolvimento de fatores de risco em idades cada vez mais precoces que, na seqüência, em idades adultas, podem manifestar-se clinicamente mediante graves disfunções cardiovasculares. De fato, estudos abordando tendência secular de indicadores biológicos associados à saúde de jovens entre 15 e 18 anos revelam acentuadas alterações desfavoráveis no perfil de lipídios/lipoproteínas plasmáticas e de pressão arterial durante última década (34).

Nessa mesma direção, estudos longitudinais envolvendo adolescentes mostram estreita associação entre estilo de vida considerado aterogênico e fato- 
res de risco predisponentes às DCV. Em ambos os sexos, componentes relacionados aos aspectos dietéticos e à prática de atividade física se caracterizam como elementos essenciais na preservação de indicadores metabólicos e funcionais direcionados à saúde cardiovascular (10).

Em vista disso, especialistas na área têm sugerido programas de intervenção direcionados a detectar eventuais agressões provenientes dos hábitos dietéticos e de prática de atividade física inadequados que possam repercutir negativamente na saúde cardio-vascular de jovens (33). Reforça-se essa posição, na medida em que padrões quanto ao estilo de vida podem ser fortemente estruturados e definidos na adolescência, e possivelmente transferidos para a idade adulta (19).

Nesse particular, ações intervencionistas podem ser implementadas mediante sofisticados procedimentos laboratoriais voltados à monitoração periódica dos fatores de risco predisponentes às DCV. No entanto, por conta do alto custo financeiro e da elevada demanda de tempo associados às rotinas de avaliação e, por vezes, tratar-se de procedimentos invasivos, torna-se inviável adotar esses procedimentos rotineiramente em grande número de jovens. Em vista disso, esforços têm sido direcionados no sentido de propor procedimentos alternativos que possam melhor atender aspectos de praticidade e de viabilidade, e que venham oferecer indicações prévias de possível presença de fatores de risco predisponentes às DCV na população jovem.

O reconhecimento de pontos de corte associados aos indicadores de aptidão física relacionada à saúde, adequados à identificação de adolescentes portadores de fatores de risco predisponentes às DCV, poderá credenciar procedimento prático e de grande acessibilidade para professores de educação física tentarem auxiliar em intervenções voltadas à progressão de fenômenos aterogênicos em idades jovens, reduzindo o risco futuro de surgimento de cardiopatias. Os resultados encontrados no estudo mostram que os indicadores morfológicos associados à aptidão física relacionada à saúde podem oferecer importantes informações quanto à eventual identificação de adolescentes portadores de fatores de risco predisponentes às DCV, sobretudo ao reduzir discretamente os pontos de corte quanto ao IMC e ao $\sum$ EDC suge- ridos na proposta Physical Best. Pontos de corte de $16-23 \mathrm{~kg} / \mathrm{m}^{2}$ para moças e $16-22 \mathrm{~kg} / \mathrm{m}^{2}$ para rapazes quanto ao IMC, e, com relação ao $\sum E D C, 16-34 \mathrm{~mm}$ e 11-24 mm para moças e rapazes, respectivamente, descreveram melhor equilíbrio entre sensibilidade e especificidade. Em valores probabilísticos, de cada quatro adolescentes que não se ajustaram aos novos pontos de corte associados ao IMC e ao $\Sigma$ EDC existem indicações de que três deles podem ser identificados como portadores de fatores de risco predisponentes às DCV.

Em razão da similaridade nos resultados das estatísticas de validação, tornando-se portanto indiferente utilizar valores de IMC ou de $\Sigma$ EDC como indicadores morfológicos na identificação da presença de fatores de risco predisponentes às DCV, chama-se atenção para o fato do $\sum E D C$ apresentar maior dificuldade de acesso as informações e mais elevada exposição aos erros de medida. O IMC é alcançado mediante razão entre o peso corporal e o quadrado da estatura, pelo que se torna mais acessível suas medidas. Sugestões de empregar um ou outro indicador morfológico na monitoração do excesso de gordura e de peso corporal associados aos agravos de saúde em populações jovens são freqüentemente encontradas na literatura $(25,27,32)$. Em assim sendo, reforça-se a posição no sentido de indicar pontos de corte equivalentes ao IMC como opção morfológica mais adequada para tentar discriminar entre adolescentes portadores e não-portadores de fatores de risco predisponentes às DCV.

De certa maneira, esses achados podem ser considerados coincidentes com estudos prévios envolvendo diferentes abordagens de análise. Mediante recursos estatísticos de regressão múltipla, verificou-se que o IMC e medidas de espessura de dobras cutâneas podem ser considerados preditores significativos de variações no perfil lipídico/lipoproteico de adolescentes (8). Em jovens entre 5 e 20 anos observou-se que os coeficientes de correlação momento-produto entre o IMC e a pressão arterial sistólica podem variar entre 0,30 e 0,40 nos rapazes e entre 0,31 e 0,57 nas moças (32).

No domínio da composição corporal, foi desenvolvido um estudo envolvendo regressão logística com intenção de apontar a quantidade de gordura relativa ao peso corporal a partir da qual os níveis de pressão 
arterial e de colesterol sérico total e frações tendiam a aumentar (42). Os resultados encontrados sugerem que moças e rapazes, ao apresentarem respectivamente $30 \%$ e $25 \%$ do peso corporal como gordura, tenderam a agregar desordens associadas ao perfil lipídico/lipoprotéico plasmático. Ao recorrer aos modelos matemáticos sugeridos por Slaughter et al. (38), direcionados à estabelecer estimativas da quantidade de gordura relativa ao peso corporal mediante espessuras de dobras cutâneas medidas nas regiões tricipital e subescapular, verifica-se aproximações bastante similares entre os limites superiores dos pontos de corte de melhor equilíbrio entre sensibilidade e especificidade (34 mm para moças e $24 \mathrm{~mm}$ para rapazes) e proporções de $30 \%$ e $25 \%$ do peso corporal como gordura.

No que se refere aos indicadores de desempenho motor da aptidão física relacionada à saúde, evidências acumuladas no estudo sugerem que os pontos de corte estabelecidos não foram suficientemente sensíveis para discriminar entre adolescentes portadores e não-portadores de fatores de risco predisponentes às DCV. Estimativas relativas às estatísticas de validação utilizadas no estudo revelaram que não mais de um a cada quatro adolescentes que apresentavam comprometimento quanto ao desempenho motor, e portanto, não alcançaram pontos de corte sugeridos na proposta Physical Best, foram efetivamente identificados como portadores de fatores de risco predisponentes às DCV. Dessa forma, ao recorrer a esses critérios na eventual identificação de adolescentes portadores de fatores de risco predisponentes às DCV, constata-se que incidência na ocorrência de casos falso-positivos e falso-negativos torna-se excessivamente elevada.

Ao procurar estabelecer pontos de corte alternativos que possam maximizar ajustes estatísticos na ocorrência de casos verdadeiros, verificou-se que, em ambos os sexos, houve necessidade de modificar significativamente os limiares dos resultados dos três testes motores sugeridos na proposta Physical Best. Contudo, ainda assim, apesar de se constatarem avanços quanto aos parâmetros de validação, as probabilidades quanto à incidência de casos falso-positivos e falso-negativos permaneceram excessivamente elevadas, ou seja, uma significativa proporção de adolescentes podem ser identificados inadequada- mente como portadores de fatores de risco predisponentes às DCV por não atenderem os pontos de corte associados aos indicadores de desempenho motor da aptidão física relacionada à saúde, e também, podem ser identificados de maneira incorreta como não-portadores de fatores de risco predisponentes as DCV por atenderem os pontos de corte associados aos indicadores de desempenho motor da aptidão física relacionada à saúde.

Em assim sendo, torna-se recomendável descartar a hipótese no sentido de que os indicadores de desempenho motor associados à aptidão física relacionada à saúde possam ser utilizados na identificação de adolescentes portadores e não-portadores de fatores de risco predisponentes às $\mathrm{DCV}$, independentemente dos pontos de corte atribuídos aos resultados dos testes motores.

De certa forma, esses resultados apresentam discordância de algumas evidências apresentadas na literatura. Baseando-se em suposta relação empírica entre níveis mais elevados de prática de atividade física e melhores índices de aptidão física, estudos anteriores comprovaram que significativa maior proporção de adolescentes mais ativos fisicamente foram capazes de atender os pontos de corte associados aos indicadores de desempenho motor sugeridos mediante proposta Physical Best $(13,28)$. Contudo, neste particular, dois aspectos devem ser considerados. Primeiro, estudos experimentais envolvendo recursos de análise de regressão mostram que informações quanto aos níveis de prática habitual de atividade física explicam somente pequena quantidade de variação dos índices de aptidão física relacionada à saúde, não garantindo necessariamente que os mais ativos são mais aptos fisicamente $(23,30)$. Segundo, apesar de se caracterizar como importante elemento contribuinte, nem sempre os jovens mais ativos fisicamente apresentam melhor estado de saúde durante a adolescência $(9,32)$.

Dessa forma, parece não ser recomendável assumir níveis de prática habitual de atividade física como critério de validação de pontos de corte de indicadores de desempenho motor associados à aptidão física relacionado à saúde, o que pode suscitar dúvidas quanto as conclusões apresentadas por estudos que recorreram a esse recurso de validação.

Possivelmente, alguns aspectos possam ser aponta- 
dos como responsáveis pela menor validação dos indicadores de desempenho motor na identificação de adolescentes portadores de fatores de risco predisponentes às DCV. De imediato, a seleção dos resultados do SEAL e do ABDO, como indicadores das condições de saúde associados ao desempenho motor, baseia-se no pressuposto de que as capacidades de flexibilidade e de força/resistência dos grupos musculares da região inferior do tronco podem ser considerados fatores importantes na prevenção e na reabilitação de eventuais lesões lombares e desvios posturais (18). Assumindo que no presente estudo o critério de validação dos pontos de corte associados aos indicadores da aptidão física relacionada à saúde refere-se às $\mathrm{DCV}$, parece razoável admitir que os itens de desempenho motor relacionados às disfunções ortopédicas possam não alcançar a validade desejada. Os pontos de corte relacionados ao CORR foram idealizados mediante informações epidemiológicas envolvendo valores estimados quanto ao consumo máximo de oxigênio e maior incidência de disfunções cardiovasculares em adultos, ajustados quanto à running economic e à idade para populações jovens (18). Desse modo, de forma antagônica ao que se observa com relação ao SEAL e ao ABDO, informações que subsidiam proposição dos pontos de corte concedidos ao CORR podem ser consideradas mais estreitamente relacionadas com os critérios de validação utilizados no estudo, qual sejam, fatores de risco predisponentes às DCV. Talvez, em vista disso é que se constatou para os pontos de corte associados ao CORR parâmetros de validação mais favoráveis, no entanto, ainda assim, distante do desejado. Além disso, mesmo considerando possíveis participações de atributos genéticos na variação dos fatores de risco predisponentes às $\mathrm{DCV}$, fundamentalmente por se tratar de adolescentes, não se pode deixar de considerar a possibilidade de ocorrência de variações nos resultados dos testes motores por conta dos níveis de habilidade apresentados pelos adolescentes na execução das tarefas motoras envolvidas na realização dos testes motores. Ainda, níveis de motivação para se alcançar esforços físicos adequados que possam traduzir resultados efetivos quanto ao real desempenho motor dos adolescentes pode se definir como outro fator de interferência na validação dos pontos de corte de indicadores de desempenho motor associados à aptidão física relacionada à saúde.

\section{CONCLUSÕES}

Os resultados encontrados no estudo sugerem que apenas os indicadores morfológicos associados à aptidão física relacionada à saúde devem ser empregados na identificação de adolescentes portadores de fatores de risco predisponentes às DCV. Os pontos de corte para o IMC e o $\sum$ EDC que oferecem melhor equilíbrio entre probabilidades de ocorrência de casos verdadeiro-positivos e falso-positivos recomendados no presente estudo são discretamente menores que os sugeridos na proposta Physical Best. Evidências acumuladas mediante desenvolvimento do estudo suportam argumentação no sentido de que, quaisquer que sejam os pontos de corte atribuídos aos indicadores de desempenho motor associados à aptidão física relacionada à saúde, esses não se definiram como aproximação metodológica adequada na identificação de adolescentes portadores de fatores de risco predisponentes às DCV.

Sugere-se o desenvolvimento de futuros estudos em diferentes grupos de adolescentes, envolvendo outros critérios de referência para saúde que não apenas fatores de risco predisponentes às $\mathrm{DCV}$, na tentativa de verificar se as deficiências quanto aos indicadores de desempenho motor associados à aptidão física relacionada à saúde podem ser confirmadas.

\author{
*Apoio do MCT/CNPq \\ CORRESPONDÊNCIA \\ Dartagnan Pinto Guedes \\ Rua Prof. Samuel Moura 328 - Apto. 901 \\ CEP. 86061-070 - Londrina \\ Paraná \\ Brasil \\ darta@sercomtel.com.br
}




\section{REFERÊNCIAS BIBLIOGRÁFICAS}

1. Almeida H; Wicherhauser PM (1991). O Critério ABA/Abipemi: em Busca de uma Atualização. São Paulo: Abipemi.

2. American Alliance for Health, Physical Education, Recreation and Dance (1984). Health Related Physical Fitness Technical Manual. Reston: American Alliance for Health, Physical Education, Recreation and Dance.

3. American Alliance for Health, Physical Education, Recreation and Dance (1988). Physical Best. Reston American Alliance for Health, Physical Education, Recreation and Dance.

4. American Health and Fitness Association (1986). Fit Young Today. Austin, Texas: American Health and Fitness Association.

5. Andersen LB; Henckel P; Saltin B (1989). Risk factors for cardiovascular disease in 16-19-year-old teenagers. Journal of Internal Medicine 225:157-63.

6. Anding JD; Kubena KS; McIntosh WA; O’Brien B (1996). Blood lipids, cardiovascular fitness, obesity and blood pressure: the presence of potential coronary heart disease risk factors in adolescents. American Journal of Diet Association 96:238-42.

7. Bell RD; Macek M; Rutenfranz J; Saris WHM (1986). Health indicators and risk factors of cardiovascular diseases during childhood and adolescence. In: Rutendranz J; Mocellin R; Klimt F (ed.). Children and Exercise XII. Champaign, Illinois: Human Kinetics Publishers, 19-27.

8. Björntorp P (1985). Obesity and the risk of cardiovascular disease. Annals of Clinical Research 17:3-9.

9. Blair SN; Clark DG; Cureton KJ; Powell KE (1989) Exercise and fitness in childhood: implications for a lifetime of health. In: Gisolfi CV; Lamb DR (ed.). Perspectives in Exercise Science and Sports Medicine: Youth, Exercise, and Sport. Indianapolis: Benchmark Press, 401-30.

10. Boreham C; Twisk J; Mechlen W; Sarage M; Strain J; Gran G (1999). Relationships between the development of biological risk factors for coronary heart disease and lifestyle parameters during adolescence: The Northern Irland Young Hearts Project. Public Health 113:7-12.

11. Bruce RA; Kusumi F; Hosmer D (1973). Maximal oxygen intake and nomographic assessment of functional aerobic impairment in cardiovascular disease. American Heart Journal 85(4):546-62.

12. Canadian Association for Health, Physical Education and Recreation (1980). The CAHPER Fitness: Performance Test Manual. Vanier: Canadian Association for Health, Physical Education and Recreation.

13. Chun DM; Corbin CB; Pangrazi RP (2000). Validation of criterion-referenced standards for the mile run and progressive aerobic cardiovascular endurance tests. Research Quarterly for Exercise and Sport 71:125-34.

14. Comité pour le Developement du Sport (1988). Manuel pour les tests EUROFIT d'Aptitude Physique. Rome: Edigraf Editoriale Grafica.

15. Cooper Institute for Aerobics Research (1999). Fitnessgram Test Administration Manual. $2^{a}$. Edition. Dallas, Texas: Cooper Institute for Aerobics Research.

16. Corbin CB; Lindsey R (1997). Concepts of Physical Fitness. $9^{\mathrm{a}}$. Edition. Dubuque: Brown \& Benchmark Publishers.

17. Corbin CB; Pangrazi RP (1992). Are American children and youth fit? Research Quarterly for Exercise and Sport 63:96-106.
18. Cureton KJ; Warren GL (1990). Criterion-referenced standards for youth health-related fitness tests: a tutorial. Research Quarterly for Exercise and Sport 61:7-19.

19. Edmundson E; Parcel GS; Feldman HA; Elder J; Perry CL; Johnson CC; Williston BJ; Stone EJ; Yang M; Lytle L; Webber L (1996). The effects of the child and adolescent trial for cardiovascular health upon psychosocial determinants of diet and physical activity behavior. Preventive Medicine 25:442-54.

20. Friedewald WT, Levy RI; Frederickson DS (1972). Estimation of the concentration of low density lipoprotein cholesterol in plasma. Without use of the preparative ultracentrifuge. Clinical Chemistry 18:499-502.

21. Guedes DP; Guedes JERP (1995). Aptidão física relacionada à saúde de crianças e adolescentes: avaliação referenciada por critério. Revista Brasileira de Atividade Física e Saúde 1:27-38.

22. Guedes DP; Guedes JERP (1997). Crescimento, Composição Corporal e Desempenho Motor de Crianças e Adolescentes. São Paulo: CLR Balieiro.

23. Guedes DP; Guedes JERP; Barbosa DS; Oliveira JA (2001) Atividade física habitual e aptidão física relacionada à saúde em adolescentes. Revista Brasileira Ciência e Movimento 10:13-21.

24. Hanley J (1988). The robustness of the "binormal" assumptions used in fitting ROC curves. Medicine Decis Making 8:197-203.

25. Himes J; Dietz W (1994). Guidelines for overweight in adolescent preventive services: recommendations from an expert committee. American Journal of Clinical Nutrition 59:307-16

26. Institute for Aerobics Research (1987). Fitnessgram User's Manual. Dallas: Institute for Aerobics Research.

27. Lazarus R; Baur L; Webb K; Blyth F (1996). Body mass index in screening for adiposity in children and adolescents: systematic evaluation using receiver operating characteristic curves. American Journal of Clinical Nutrition 63:500-6.

28. Looney MA; Plowman SA (1990). Passing rates of American children and youth on the fitnessgram criterionreferenced physical fitness standards. Research Quarterly for Exercise and Sport 61:215-22.

29. Malina RM; Bouchard C (1991). Growth, Maturation, and Physical Activity. Champaign, Illinois: Human Kinetics Publishers Inc.

30. Morrow JR; Freedson PS (1994). Relationships between habitual physical activity and aerobic fitness in adolescents. Pediatric Exercise Science 6:315-29.

31. MedCalc (1997). Computer Program for Statistics in Medicine for Windows: Program Manual. Mariakerke, Belgium: MedCalc Software.

32. Nicklas TA; Webber LS; Johnson CC; Srinivasan SR Berenson GS (1995). Foundations for health promotion with youth: a review of observations from the Bogalusa Heart Study. Journal of Health Education 26:S18-S26.

33. Pate RR; Hohn RC (1994). Health and Fitness through Physical Education. Champaign, Illinois: Human Kinetics.

34. Porkka KVK; Raitakari OT; Leino A; Laitinen S; Rasanen L; Ronnemaa T; Marniemi J; Lehtimaki T; Taimela S; Dahl M; Uhari M; Akerblom HK; Viikari JSA (1997). Trend in serum lipid levels during 1980-1992 in children and young adults. American Journal of Epidemiology 146:64-77. 
35. President's Council on Physical Fitness and Sports (1997). 1996-97 The President's Challenge Physical Fitness Program Packet. Bloomington: Poplars Research Center.

36. Raitakari OT; Porkka KVK; Viikari JSA; Rönnemaa T; Akerblom HK (1994). Clustering of risk factors for coronary heart disease in children and adolescents. The Cardiovascular Risk in Young Finns Study. Acta Paediatrica 83:935-40

37. Schmidt GJ; Walkuski JJ; Stensel DJ (1998). The Singapore youth coronary risk and physical activity study. Medicine and Science in Sport and Exercise 30:105-13.

38. Slaughter MH; Lohman TG; Boileau RA; Horswill CA; Stillman RJ; Van Loan MD; Benben DA (1988). Skinfold equations for estimation of body fatness in children and youth. Human Biology 60:709-23.

39. Tanner JM (1962). Growth at Adolescence. 2a. Edition. Oxford: Blackwell Scientific Publications.

40. Varrasi G; Bazzano C (1990). A comparison of health-related fitness of central Italians and American children. In: Hermans GPH; Mosterd WL (ed.). Sports, Medicine and Health. Amsterdam: Excerpta Medica, 967-72.

41. Weiller KH; Jackson AW; Meyer RD (1994). 1-Mile run performance and body mass index in hispanic youth: passing rates for the fitnessgram. Pediatric Exercise Science 6:267-74.

42. Williams DP; Going SB; Lohman TG; Harsha DW; Srinivasan SR; Webber LS; Berenson GS (1992). Body fatness and risk for elevated blood pressure, total cholesterol, and serum lipoprotein ratios in children and adolescents. American Journal of Public Health 82:358-63.

43. Zweig MH; Campbell G (1993). Receiver-operating characteristic (ROC) plots: a fundamental evaluation tool in clinical medicine. Clinical Chemistry 39:561-77. 